\title{
Etude de la diffraction de la houle par un caisson Jarlan
}

\section{Catherine Fleury}

Elève Ingénieur à l'Ecole Supérieure d'Ingénieurs de Marseille

\author{
Mamoun Naciri \\ Département Génie Côtier, OCEANIDE.
}

\section{Résumé}

La diffraction d'onde de gravité en incidence normale sur des caissons rectangulaires semi-immergés est étudiée théoriquement. Le modèle numérique bidimensionnel est basé sur la théorie des potentiels linéarisés. Cette étude a porté sur un caisson de type "Jarian" doté de plusieurs chambres d'atténuation (murs perforés). Des équations de pertes de charge à travers ces murs perforés ont été utilisés. Le modèle numérique a été comparé à des données expérimentales et il a pu ainsi être validé. L'attrait de ce modèle est qu'il permettra d'optimiser ce type d'amortisseur de houle préalablement à la conduite d'essais en modèle réduit.

\section{Introduction}

La construction de digues en grande profondeur devient une nécessité pour l'extension de port sur des littoraux accores comme ceux de la Méditerranée française orientale. Il est impossible ou économiquement peu rentable d'envisager, dans ce contexte, des solutions classiques telles que la digue à talus ou la digue verticale. Par contre, des solutions dérivées de techniques éprouvées et communément utilisées dans le domaine offshore sont bien adaptées aux profondeurs de l'ordre de $50 \mathrm{~m}$. Ces profondeurs, considérées comme importantes pour le concepteur de ports, sont faibles pour le concepteur offshore. Ces techniques sont fondées sur l'utilisation de derricks (jackets en terminologie offshore) ancrés dans le sol au moyen de pieux et sur lesquels viennent se fixer des caissons en béton semi-immergés (voir figure 1). Le principe de base est celui du mur d'eau fixe. Compte tenu de la répartition dans la colonne d'eau de l'énergie véhiculée par la houle, de faibles coefficients de transmission peuvent être obtenus pour un tirant d'eau égal à une fraction de la profondeur totale.

Afin de diminuer les efforts horizontaux en tête qui sont souvent dimensionnants, de minimiser le coefficient de réflexion du caisson et l'agitation résiduelle derrière celui-ci, des chambres de dissipation dotées de murs perforés (type Jarlan) sont disposées sur les faces amont et aval du caisson.

Des digues de protection de ce type sont à l'étude actuellement pour l'extension des ports de Nice et Monaco.

L'intérêt de disposer d'une formulation théorique du problème de la diffraction vient de la possibilité d'effectuer à moindre coût le prédimensionnement de tels ouvrages avant d'aborder l'étape de validation par des essais en canal à houle. 


\section{Bibliographie}

Jarlan (1965), par analogie avec le domaine de l'acoustique, a le premier introduit le concept de la paroi perforée assortie d'une chambre de dissipation comme moyen d'amorissement d'ondes de gravite. Comme exemple d'application de ce concept, on peut citer l'enceinte de protection annulaire de $92 \mathrm{~m}$ de diamètre construite autour de la plateforme Ekofisk en mer du Nord. Motivés par plusieurs projets de ponts semi-immergés traversant Lake Washington (Washington, Etats-Unis), Richey et al (1970) ont analysés le fonctionnement hydraulique d"une chambre de dissipation sous l'attaque d'une houle avec pour objectif principal la minimisation du coefficient de réflexion. Avec l'hypothèse simplificatrice d'une chambre dont la surface libre reste toujours horizontale, et une linearisation de type Lorentz, les auteurs aboutissent à une équation différentielle du second ordre régissant le niveau d'eau dans la chambre. Des considérations énergétiques conduisent ensuite à une expression du coefficient de réflexion en fonction des paramètres géométriques du système. L'analyse de Richey et al ne met pas l'accent sur la transmission et ne considère pas la modification de la houle par la présence d'un obstacle semiimmergé. A la même époque, Mei et al (1969) ont étudié la diffraction d'ondes de gravité par un obstacle situé soit à la surface soit au fond. Une méthode variationnelle assortie de considérations de symétrie est utilisée. Des coefficients de réflexion et de transmission complexes sont obtenus numériquement. Ce problème a été étudié plus récemment par Johansson (1989) et Rey (1995) en utilisant une décomposition en modes propres dans chaque domaine et des propriétés d'orthogonalité. Si Jarlan a introduit l'idée de parois perforées, ce n'était pas seulement pour des raisons hydrauliques, mais également et peut être surtout dans loptique de diminuer les efforts exercés par les particules d'eau en mouvement. $\mathrm{Ce}$ type d'argument a conduit notamment Molin (1992-1993) à proposer des semisubmersibles (offshore) constitués de cylindres perforés et le percement des plaques atténuatrices de houle. Dans les deux cas, la perte de charge à traverș une paroi perforée est́ donnée par $\Delta p=\rho \frac{1-\tau}{2 \mu \tau^{2}} V|V|$ où $\tau$ désigne la porosité, $\mu$ un paramètre géométrique proche de 1 et $\mathrm{V}$ la vitesse des particules d'eau à travers les perforations. Fourest (1991) a étudié l'atténuation de la houle par une série de plaques perforées disposées verticalement de la surfacé libre au fond avec pour application une utilisation comme système d'amortissement en bout de canal à houle. La formule de perte de charge précédente est utilisée. Des essais en modèle réduit ont été effectués à plusieurs reprises à OCEANIDE dans le but de mesurer les caractéristiques hydrauliques de caissons munis de chambres de dissipation. Certains résultats seront utilisés pour comparaison avec les résultats numériques.

\section{Modélisation}

\subsection{Hypothèses}

Les hypothèses retenues pour la modélisation de la diffraction d'une onde de gravité par un caisson Jarlan sont maintenant énumérées :

- Modélisation bi-dimensionnelle dans le plan vertical,

- Fluide parfait, 
- Ecoulement potentiel dans chaque domaine (hypothèse d'irrotationnalité),

- Onde de gravité monochromatique en incidence normale,

- Théorie linéaire d'Airy,

- Absence de franchissement des parois perforées par la houle,

- Formule de perte de charge à travers une paroi perforée quadratique.

\subsection{Principes de modélisation}

La figure 1 ci-dessous illustre la manière dont le fluide a été décomposé en plusieurs domaines numérotés de 1 à 6 .

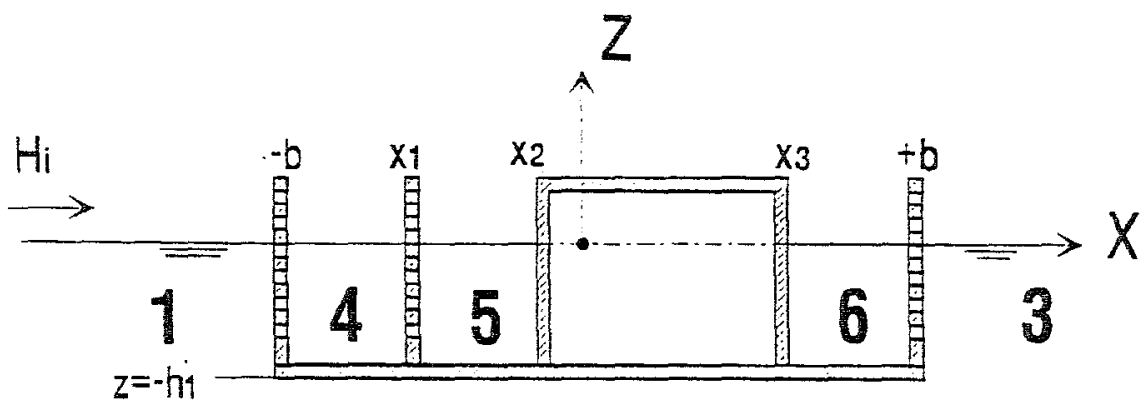

\section{2}

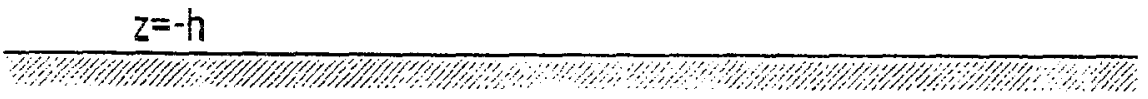

Figure 1 - Schéma de principe de la modélisation.

Avec les hypothèses précédentes, le potentiel complexe se présente sous la forme

$\Phi(\mathrm{x}, \mathrm{z}, \mathrm{t})=\varphi(\mathrm{x}, \mathrm{z}) \mathrm{e}^{-\mathrm{i} \omega \mathrm{t}}$

La procédure utilisée consiste à écrire de façon générale l'expression des potentiels $\varphi_{1}$ à $\varphi_{6}$ et de satisfaire ensuite des conditions de raccord à la frontière des différents domaines.

\subsubsection{Formulation des potentiels}

Dans les domaines 1 et 3 , la profondeur est égale à $h$. Dans le domaine 2, elle vaut $\mathrm{h}-\mathrm{h}_{1}$. Enfin, la profondeur d'eau dans les chambres est égale à $\mathrm{h}_{1}$.

Le potentiel des domaines 1 et 3 s'écrit comme une combinaison linéaire de modes propres :

$\varphi_{1}(x, z)=\frac{\cosh k(z+h)}{\cosh \cdot k h}\left(A_{i} e^{i k x}+R e^{-i k \cdot(x+b)}\right)+\sum_{p=1}^{n} c_{l p} e^{k_{p}(x+b)} \frac{\cos k_{p}(z+h)}{\cos k_{p} h}$

$\varphi_{3}(x, z)=\frac{\cosh . k(z+h)}{\cosh \cdot k h} T e^{i k(x-b)}+\sum_{p=1}^{n} d_{3 p} e^{-k_{p}(x+b)} \frac{\cos k_{p}(z+h)}{\cos k_{p} h}$ 
Chacun de ces potentiels comporte une composante propagative (ondes incidente et réfléchie pour le domaine 1 et transmise pour le domaine 3 ) de vecteur dionde $k$ et une sommation d'ondes évanescentes de vecteur d'onde $k_{\mathrm{p}}$. Ces vecteurs d'onde vérifient les relations de dispersion suivantes :

$\omega^{2}=g k \tanh (\mathrm{kh})$

pour les modes propagarifs

$\omega^{2}=-g k_{p} \tan \left(k_{p} h\right) \quad p=1, \ldots, n$

pour les modes évanescents, avec $\omega=2 \pi / \mathrm{T}$.

Le potentiel du domaine 2 quant à lui s'écrit

$\omega_{2}(x, z)=\frac{P}{b} x+Q+\sum_{p=1}^{n}\left(c_{2 p} e^{-k_{p}^{\prime}(x+b)}+d_{2 p} e^{k_{p}^{\prime}(x-b)}\right) \cos k_{p}^{\prime}(z+h)$

où les vecteurs d'onde $\mathrm{k}_{\mathrm{p}}^{\prime}$ satisfont la relation

$k_{p}^{\prime}=p \pi /\left(h-h_{1}\right)$

Le potentiel dans chacune des trois chambres s'écrit de la façon suivante

$\varphi_{4}(x, z)=\frac{\cosh K\left(z+h_{1}\right)}{\cosh K_{1}}\left(\alpha e^{i K .}+\beta e^{-i K x}\right)$

$\varphi_{5}(\mathrm{x})=\gamma \cos \mathrm{K}\left(\mathrm{x}-\mathrm{x}_{2}\right)$

$\varphi_{6}(\mathrm{x})=\delta \cos \mathrm{K}\left(\mathrm{x}-\mathrm{x}_{3}\right)$

où le nombre d'onde $\mathrm{K}$ satisfait la relation de dispersion

$\omega^{2}=\mathrm{gK} \tanh \left(\mathrm{Kh}_{1}\right)$

Les modes évanescents ont été omis de l'expression de $\varphi_{4}$ par simplicité. Une approximation de type ondes longues est utilisée pour $\varphi_{5}$ et $\varphi_{6}$.

La forme des potentiels $\varphi_{5}$ et $\varphi_{6}$ est telle que les concitions d'imperméabilité sur les parois pleines sont satisfaites. Si n est le nombre de modes évanescents utilisés dans les sommations précédentes, il y a $8 n+8$ inconnues complexes.

\subsubsection{Conditions de raccord}

Des conditions de raccord doivent être satisfaites à la frontière entre deux domaines adjacents. Ces conditions (continuité des vitesses et des pressions où la spécification de pertes de charge à travers une paroi perforée) sont satisfaites au sens faible en annulant leurs projections: sur une base de fonctions propres orthogonales. On utilise pour cela les fonctions propres suivantes :

$\chi_{1,3}(z)=\cosh k(z+h) \quad \Psi_{1,3 p}(z)=\cos k_{p}(z+h) \quad p=1, \ldots, n$

pour les domaines 1 et 3 et

$\chi_{2}(z)=1 \quad \Psi_{2 p}(z)=\cos k_{p}^{\prime}(z+h) \quad p=1, \ldots, n$ 
pour le domaine 2 . Les pertes de charge à travers une paroi perforée s'expriment en fonction des potentiels $\varphi_{\mathrm{g}}$ et $\varphi_{\mathrm{d}}$ à gauche et à droite de cette paroi de la façon suivante :

$\varphi_{\mathrm{g}}-\varphi_{\mathrm{d}}=-\mathrm{i} \frac{4(1-\tau)}{3 \pi \mu \tau^{2} \omega}\left\|\varphi_{\mathrm{gx}}\right\| \varphi_{\mathrm{gx}}$

où $\tau$ désigne le taux de perforation (ratio de la surface des perforations sur la surface totale). Le coefficient $8 / 3 \pi$ qui apparait dans (3.5) provient de la linéarisation en temps de Lorentz.

$A x=-b$, les conditions suivantes sont satisfaites

$$
\begin{aligned}
& \int_{-\mathrm{h}}^{0} \varphi_{1 \mathrm{x}} \chi_{1,3} \mathrm{dz}=\int_{-\mathrm{h}}^{-\mathrm{h}_{1}} \varphi_{2 \mathrm{x}} \chi_{1,3} \mathrm{~d} z+\int_{-\mathrm{h}_{1}}^{0} \varphi_{4 \mathrm{x}} \chi_{1,3} \mathrm{dz} \\
& \int_{-\mathrm{h}}^{0} \varphi_{1 \mathrm{x}} \Psi_{1,3 \mathrm{p}} \mathrm{dz}=\int_{-\mathrm{h}}^{-\mathrm{h} 1} \varphi_{2 \mathrm{x}} \Psi_{1,3 \mathrm{p}} \mathrm{d} z+\int_{-\mathrm{h}_{1}}^{0} \varphi_{4 \mathrm{x}} \Psi_{1,3 \mathrm{p}} \mathrm{dz} \quad \mathrm{p}=1, \ldots, \mathrm{n} \\
& \int_{-\mathrm{h}}^{-\mathrm{h}_{1}} \varphi_{1} \chi_{2} \mathrm{dz}=\int_{-\mathrm{h}}^{-\mathrm{h}_{1}} \varphi_{2} \chi_{2} \mathrm{~d} z \quad \int_{-\mathrm{h}}^{-h_{1}} \varphi_{1} \Psi_{2, \mathrm{p}} \mathrm{d} z=\int_{-\mathrm{h}}^{-\mathrm{h}_{1}} \varphi_{2} \Psi_{2, \mathrm{p}} \mathrm{dz} \quad \mathrm{p}=1, \ldots, \mathrm{n} \\
& \int_{-\mathrm{h}_{1}}^{0}\left[\varphi_{1}-\varphi_{4}+\mathrm{i} \frac{4(1-\tau)}{3 \pi \mu \tau^{2} \omega}\left\|\varphi_{1 \mathrm{x}}\right\| \varphi_{1 \mathrm{x}}\right] \chi_{1,3} \mathrm{dz}=0 \\
& \int_{-\mathrm{h}_{1}}^{0}\left[\varphi_{1}-\varphi_{4}+\mathrm{i} \frac{4(1-\tau)}{3 \pi \mu \tau^{2} \omega}\left\|\varphi_{1 \mathrm{x}}\right\| \varphi_{1 \mathrm{x}}\right] \Psi_{1,3 \mathrm{p}} \mathrm{dz}=0 \quad \mathrm{p}=1, \ldots, \mathrm{n}
\end{aligned}
$$

$(3,6 a)$ traduit la continuité du débit, $(3,6 b)$ celle de la pression sous le caisson et $(3,6 c)$ traduit les pertes de charge. En $x=+b$, autant de conditions sont satisfaites.

En $\mathrm{x}=\mathrm{x}_{1}$, la continuité des débits donne

$$
\int_{-\mathrm{h}_{1}}^{0} \varphi_{4 \mathrm{x}} \chi_{1,3} \mathrm{dz}=\int_{-\mathrm{h}_{1}}^{0} \varphi_{5 \mathrm{x}} \chi_{\mathrm{L}, 3} \mathrm{dz} \quad \int_{-\mathrm{h}_{1}}^{0} \varphi_{4 \mathrm{x}} \Psi_{1,3 \mathrm{p}} \mathrm{dz}=\int_{-\mathrm{h}_{1}}^{0} \varphi_{5 \mathrm{x}} \Psi_{1,3 \mathrm{p}} \mathrm{dz} \quad \mathrm{p}=1, \ldots, \mathrm{n}
$$

et la spécification des pertes de charge donne une formule similaire à $(3.6 \mathrm{e}, \mathrm{f})$. Au total, $8 \pi+8$ conditions sont exprimées pour autant d'inconnues.

\subsection{Méthode de résolution}

L'équation non-linéaire de perte de charge à travers les parois perforées nécessite un schéma de résolution itératif. Les modalités de résolution du système linéaire sont évoquées au $\$ 3.3 .1$ et le critère de convergence au $\S 3.3 .2$.

\subsubsection{Inversion}

A chaque itération, un système linéaire de $8 n+8$ inconnues complexes doit être inversé ; $n$ désignant le nombre de modes évanescents pris en compte. La méthode LU de résolution est utilisée. Afin de tirer parti de l'algorithme de Numerical Recipes, le système d'équations est mis sous forme réelle.

\subsubsection{Convergence}

L'équation de perte de charge est linéarisée de la façon suivante à l'itération j: 
où le coefficient $\mathrm{c}(\mathrm{j}-1)$ est calculé à litération précédente. Les $\mathrm{c}(\mathrm{j})$ sont définis par :

$c^{(j)}=-i \frac{4(1-\tau)}{3 \pi \mu \tau^{2} \omega}\left\|\frac{\partial \varphi_{g}}{\partial x}\right\|^{(j)}$

La convergence est acquise quand la condition suivante est satisfaite

$\left\|\left(c^{(j)}-c^{(j-1)}\right) / c^{(j)}\right\|<\xi$

avec $\xi$ un petit paramètre.

\section{Validations}

Afin de valider le modèle numérique, deux cas particuliers ont été étudiés : la diffraction par un caisson plein et une approche ondes longues du caisson Jarian.

\subsection{Caisson plein}

Le domaine fluide est maintenant décomposé en trois sous-domaines (ceux précédemment numérotés 1,2 et 3 ). L'expression des potentiels $\varphi_{1}, \varphi_{2}$ et $\varphi_{3}$ est reconduite. Le nombre d'inconnues complexes est $4 n+4$ en considérant $n$ modes évanescents.

Les conditions de raccord en $-b$ et $+b$ sont récapitulées

(a) $x=-b$

$\int_{-h}^{0} \varphi_{1 x} \chi_{1,3} d z=\int_{-h}^{-h_{1}} \varphi_{2 x} \chi_{1,3} d z \quad$ et $\int_{-h}^{0} \varphi_{1 x} \Psi_{1,3 p} d z=\int_{-h}^{-h_{1}} \varphi_{2 x} \Psi_{1,3 p} d z \quad p=1, \ldots, n$

$\int_{-h}^{-h_{1}} \varphi_{1} \chi_{2} d z=\int_{-h}^{-h_{1}} \varphi_{2} \chi_{2} d z$ et $\int_{-h}^{-h_{1}} \varphi_{1} \Psi_{2 p} d z=\int_{-h}^{-h_{1}} \varphi_{2} \Psi_{2 p} d z \quad p=1, \ldots, n$

et $(a)=b$

$$
\begin{aligned}
& \int_{-\mathrm{h}}^{0} \varphi_{3 \mathrm{x}} \chi_{1,3} \mathrm{~d} z=\int_{-\mathrm{h}}^{-\mathrm{h}_{1}} \varphi_{2 \times} \chi_{1,3} \mathrm{~d} z \text { et } \int_{-\mathrm{h}}^{0} \varphi_{3 \mathrm{x}} \Psi_{1,3 \mathrm{p}} \mathrm{d} z=\int_{-\mathrm{h}}^{-\mathrm{h}_{1}} \varphi_{2 \mathrm{x}} \Psi_{1,3 \mathrm{p}} \mathrm{d} z \quad \mathrm{p}=1, \ldots, \mathrm{n} \\
& -\mathrm{h}_{1} \varphi_{3} \chi_{2} \mathrm{~d} z=\int_{-\mathrm{h}}^{-\mathrm{h}_{1}} \varphi_{2} \chi_{2} \mathrm{~d} z \text { et } \int_{-\mathrm{h}}^{-h_{1}} \varphi_{3} \Psi_{2 \mathrm{p}} \mathrm{d} z=\int_{-\mathrm{h}}^{-\mathrm{h}_{1}} \varphi_{2} \Psi_{2 \mathrm{p}} \mathrm{dz} \quad \mathrm{p}=1, \ldots, \mathrm{n}
\end{aligned}
$$

La conservation de l'énergie, condition nécessaire mais non suffisante, a été vérifiée à $10^{-5}$ près pour les périodes $6 \mathrm{~s}$ à $12 \mathrm{~s}$ qui sont généralement rencontrées en Méditerranée.

Ensuite, les résultats obtenus ont été comparés à ceux de Rey (1995) pour un caisson de $7 \mathrm{~m}$ de tirant d'eau, de $42 \mathrm{~m}$ de large par fonds de $60 \mathrm{~m}$. L'accord est très concluant comme en témoigne la figure 2 ci-après.

\subsection{Méthodes des ondes longues}

Cette approche simplificatrice consiste à relaxer la dépendance des potentiels vis-àvis de la coordonnées verticale $z$. L'expression des potentiels devient :

$\varphi_{1}(x)=A e^{i k x+R e^{-i k(x+b)}}$ 


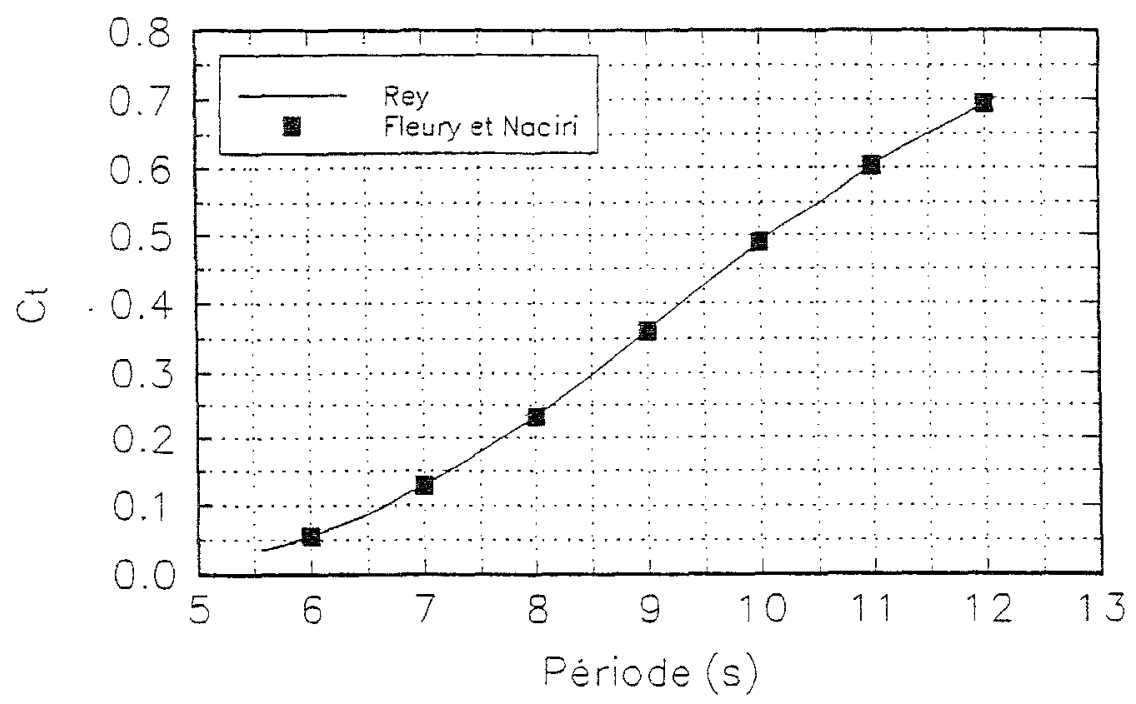

Figure 2 - Comparaison de coefficients de transmission.

$\varphi_{2}(\mathrm{x})=\frac{\mathrm{p}}{\mathrm{b}} \mathrm{x}+\mathrm{Q}$

$\varphi_{3}(x)=T e^{i k(x-b)}$

$\varphi_{4}(\mathrm{x})=\alpha \mathrm{e}^{\mathrm{ikx}}+\beta \mathrm{e}^{-\mathrm{ikx}}$

$\varphi_{5}(\mathrm{x})=\gamma \cos \mathrm{K}\left(\mathrm{x}-\mathrm{x}_{2}\right)$

$\varphi_{6}(\mathrm{x})=\delta \cos \mathrm{K}\left(\mathrm{x}-\mathrm{x}_{3}\right)$

Les conditions de raccordement se simplifient de la manière suivante :

$\varphi_{1}=\varphi_{2} @ \mathrm{x}=-\mathrm{b} \quad \varphi_{3}=\varphi_{2} @ \mathrm{x}=\mathrm{b}$

$\mathrm{h} \frac{\partial \varphi_{1}}{\partial \mathrm{x}}=\left(\mathrm{h}-\mathrm{h}_{1}\right) \frac{\partial \varphi_{2}}{\partial \mathrm{x}}+\mathrm{h}_{1} \frac{\partial \varphi_{4}}{\partial \mathrm{x}} @ \mathrm{x}=-\mathrm{b}$

$h \frac{\partial \varphi_{3}}{\partial x}=\left(h-h_{1}\right) \frac{\partial \varphi_{2}}{\partial x}+h_{1} \frac{\partial \varphi_{6}}{\partial x} @ x=+b$

$\frac{\partial \varphi_{4}}{\partial x}=\frac{\partial \varphi_{5}}{\partial x} @ x=x_{1}$

Les équations $(4.3 \mathrm{a}-\mathrm{b})$ traduisent la continuité des pressions, $(4.3 \mathrm{c}, \mathrm{e})$ la continuité des débits. Enfin, l'équation de perte de charge (3.5) est appliquée à $x=-b, x=x_{1}$ et $\mathrm{x}=\mathrm{b}$. La méthode de résolution est identique à celle décrite au $\S 3$. Des calculs ont ensuite été effectués pour un large intervalle de période ; l'objectif étant qu'aux grandes périodes l'hypothèse "ondes longues" soit réaliste. La géométrie utilisée est particulière car le tirant d'eau est égal à la profondeur $(7 \mathrm{~m})$. Les deux chambres de dissipation ont pour largeur $10 \mathrm{~m}$ et $6 \mathrm{~m}$ et les parois sont perforées à $40 \%$ et $20 \%$ d'amont en aval. Les résultats ont également été comparés avec ceux de Scolan (1995) à partir d'un modèle numérique dérivé de Fourest (1991). Les résultats de cette comparaison sont illustrés sur la figure 3 après. 


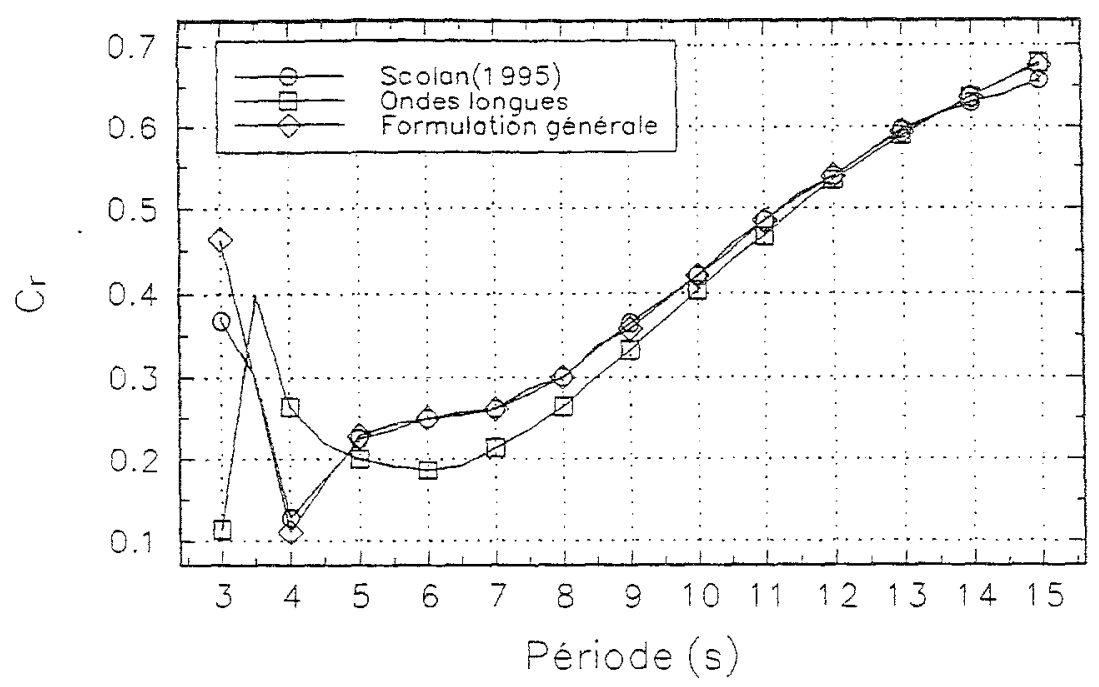

Figure 3 - Comparaison de coefficients de réfexion.

On note à toutes les périodes, un très bon accord entre Scolan (1995) et la formulation générale. Pour les faibles périodes, un écart important est observé entre ces résultats et la formulation "ondes longues". Cet écart était prévisible compte tenu de la non satisfaction de la condition "ondes longues" pour les petites périodes. Pour les périodes supérieures à $7 \mathrm{~s}$ par contre, les résultats obtenus avec et sans hypothèse "ondes longues" sont d'autant plus proches que la période augmente.

\section{Résultats}

Les résultats du modèle numérique ont été comparés avec les coefficients de réflexion et de transmission obtenus au cours d'une campagne d'essais réalisée pour le compte de DORIS ENGINEERING. Un bon accord est observé entre résultats numérique et expérimentaux. D'autres validations et comparaisons sont actuellement en cours à partir d'anciennes campaggnes d'essais effectuées à OCEANIDE.

\section{Conclusions}

Un modèle numérique est développé pour décrire, dans le cas bidimensionnel, le fonctionnement hydraulique d'un caisson semi-immergé muni de chambres de dissipation en amont et en aval. Le modèle a fait l'objet de validations en considérant, d'une part, un caisson plein ế, d'autre part, en faisant une hypothèse de type "ondes longues". Par ailleurs, la comparaison de résultats numériques et expérimentaux donne des éléments encourageants qui doivent être confirmés grâce à d'autres campagnes d'essais. L'un des objectifs. recherché est d'analyser l'influence de chacun des nombres adimensionnels dont dépendent les coefficients de réflexion et de transmission. Un autre objectif est la validation du cas limite $\tau=1$ (paroi transparente) qui permet de simuler un caisson doté d'un becquet. 


\section{Références}

Fourest J.M. 1991, Etude de la modélisation d'un amortisseur de houle constitué de plaques poreuses verticales, Rapport de stage effectué à l'IFP.

Jarlan G.L.E. 1965, The application of Acoustic Theory to the Reflective Properties of Coastal Engineering Structures. National Research Council of Canada Report No DME/NAE pp 23-63.

Johansson M. 1989, Barrier-type breakwaters, Transmission, reflection and forces. Report Series A : 19, Chalmers University of Technology, Göteborg, Sweden.

Mei C.C. and J.L. Black 1969, Scattering of surface waves by rectangular obstacles in waters of finite depth J. Fluid Mech, vol 38 pp 499-511.

Molin B. 1992, A New, Small Semi Concept for Marginal Fields, Proceedings of 24th annual Offshore Technology Conference.

Molin B. 1993, Atténuation de la houle par une dalle horizontale immergée et perforée, 4ème journée de l'hydrodynamique.

Press W.H., S.A. Teukolsky, W.T. Vetterling and B.P. Flannery 1992, Numerical Recipes in Fortran, Second Edition Cambridge University Press.

Rey V. 1995, A note on the scattering of obliquely incident surface gravity waves by cylindrical obstacles in waters of finite depth Eur J. Mech. B/Fluids, $14 \mathrm{n}^{\circ} 2$, 207-216.

Richey E. P. and C. K. Sollitt 1970, Wave attenuation by porous walled breakwater. $J$. of the Waterways, Harbors and Coastal Engineering Division, vol 96, No ww3, PP 643-663.

Scolan Y.M. 1995, Communication privée. 